\title{
Benign Solitary Fibrous Histiocytoma of Xanthomatous Subtype of the Perilimbal Conjunctiva and Adjacent Sclera in a Youth
}

\author{
Andre Ali-Ridha ${ }^{a, b}$ Seymour Brownstein ${ }^{a, b}$ Michael O'Connor ${ }^{a, c}$ \\ Tatyana Milman ${ }^{d}$ Tina Tang ${ }^{a, b}$ \\ a Department of Ophthalmology, University of Ottawa, The Ottawa Hospital, Ottawa, ON, Canada; \\ ${ }^{b}$ Department of Pathology, University of Ottawa, The Ottawa Hospital, Ottawa, ON, Canada; \\ 'Children's Hospital of Eastern Ontario, Ottawa, ON, Canada; ${ }^{d}$ Departments of Ophthalmology and \\ Pathology, Wills Eye Hospital, Philadelphia, PA, USA
}

\section{Established Facts}

- Most ocular histiocytic tumors are fibrous histiocytomas located in the orbit.

- Fibrous histiocytomas contain fibrous tissue, frequently in a storiform pattern, and variable numbers of multinucleated histiocytes.

\section{Novel Insights}

- A lesion of numerous foamy histiocytes without multinucleated giant cells or a storiform configuration in the perilimbal conjunctiva and adjacent sclera of a youth is a rare variant of fibrous histiocytoma of the xanthomatous subtype.

\section{Keywords}

Xanthoma, solitary · Fibrous histiocytoma, benign .

Excision · Ocular histiocytic tumor $\cdot$ Conjunctiva $\cdot$ Sclera

\section{Abstract \\ Aims: To report the clinical and pathological features of a benign fibrous histiocytoma of the xanthomatous subtype in the perilimbal conjunctiva and adjacent sclera in a youth. Methods: An 11-year-old Caucasian boy presented with a yellowish dome-shaped conjunctival mass abutting the in-}

ferotemporal limbus of the left eye. The tumor measured 4 $\mathrm{mm}$ in its maximum diameter. The lesion was excised and was noted to extend into the sclera. Three years postoperatively, there was no evidence of recurrence. Results: Histopathological examination disclosed a highly cellular lesion composed predominantly of benign-appearing foamy histiocytes without multinucleated giant cells. The tumor contained sparse fibrous septa without a storiform configuration. Immunohistochemical analysis showed diffuse cytoplasmic positive staining with adipophilin, CD34, and CD163. Conclusion: A lesion comprised of numerous homo-

\section{KARGER}

(C) 2018 S. Karger AG, Basel

E-Mail karger@karger.com

www.karger.com/oop
Seymour Brownstein, MD, FRCSC

The Ottawa Hospital, General Campus

501 Smyth Road, Suite W6213

Ottawa, ON K1H 8L6 (Canada)

E-Mail sbrownstein@ohri.ca 
geneous foamy histiocytes without multinucleated giant cells in the perilimbal conjunctiva and adjacent sclera of a youth is presented as a unique case of a rare variant of fibrous histiocytoma of the xanthomatous subtype.

(C) 2018 S. Karger AG, Basel

\section{Introduction}

Ocular histiocytic tumors comprise a spectrum of morphological variants, including juvenile xanthogranuloma (JXG), Langerhans cell histiocytosis (LCH), and sinus histiocytosis with massive lymphadenopathy (RosaiDorfman disease) [1]. JXG typically shows histologic evidence of histiocytes with foamy cytoplasm and Toutontype giant cells [2]. LCH is characterized as a proliferation of Langerhans-type cells with indented grooved nucleus [3]. LCH expresses CD1a markers and has characteristic Birbeck granules on electron microscopic examination [3]. Rosai-Dorfman disease involves a process of emperipolesis and cells that stain S-100 positive [1]. The most common histiocytic tumor located in the orbit is the fibrous histiocytoma, which contains a prominent fibrous component frequently with a storiform pattern [4]. We report in a youth a unique presentation of a rare variant of fibrous histiocytoma of the xanthomatous subtype of the perilimbal conjunctiva and adjacent sclera that was composed almost exclusively of foamy lipid-laden histiocytes with minimal intervening fibrous tissue and no evidence of multinucleated giant cells.

\section{Case Report}

A healthy 11-year-old Caucasian boy presented with an asymptomatic, stable, non-tender, yellowish, dome-shaped conjunctival mass abutting the inferotemporal limbus of the left eye (Fig. 1a). His parents indicated that the lesion was present for at least 1 year. The mass measured almost $4 \mathrm{~mm}$ in maximum diameter and contained several fine blood vessels. Ultrasound biomicroscopic examination disclosed a maximum thickness of $1.06 \mathrm{~mm}$, with extension to within $0.5 \mathrm{~mm}$ of the anterior chamber (Fig. 1b). The remaining ophthalmologic examination was unremarkable. There were no cutaneous lesions and no history of ocular trauma. The lesion was excised and was noted to extend into the sclera but a scleral patch graft was not required. Three years postoperatively, there was no evidence of recurrence, and examination, including gonioscopy and ultrasound biomicroscopy, was normal and unchanged from that noted at the 1-year follow-up (Fig. 1c, d).

Histopathological examination disclosed a highly cellular lesion, extending to the deep surgical margins, composed of numerous histiocytes, which had a foamy cytoplasm and benign-appearing nuclei (Fig. 2a, b). The mass contained small blood vessels in sparse fibrous septa without a storiform configuration. Lymphocytes and rare eosinophils were noted. Multinucleated giant cells were not identified.

Immunohistochemical analysis showed diffuse cytoplasmic positive staining with adipophilin (Fig. 2c), CD68, CD163 (Fig. 2d), factor XIIIa, vimentin, CD34, and CD45. Staining for S100, melan$\mathrm{A}$, and CD1a was negative. The Ki67 stain was positive in $15 \%$ of cells. These findings were most consistent with a diagnosis of a rare variant of fibrous histiocytoma of xanthomatous subtype [5].

\section{Discussion}

The clinical differential diagnosis included a lipodermoid, JXG, and fibrous histiocytoma of a common fibrous histological type. Lipodermoids on histopathological examination contain abundant adipose tissue and usually some epidermal appendages including hair follicles and sebaceous glands, which were not present in our patient's lesion. Typical fibrous histiocytomas contain variable numbers of spindled fibroblasts with fibrous tissue, frequently in a storiform pattern, and of heterogeneous histiocytes, usually including multinucleated giant cells [6-9]. In contrast, our case contained a homogenous distribution of xanthomatous histiocytes with sparse fibrous septa without a storiform configuration or multinucleated giant cells. Although the immunohistopathological profile for fibrous histiocytoma is highly variable, several reports indicate fibrous histiocytoma to stain positive for factor XIIIa for dendritic cells, vimentin for mesenchyme tissue, and CD68 for macrophages, and to stain negative for S100, CD34, and CD1a [8-10]. This profile is consistent with our case's immunohistopathological profile, except for our positive CD34. Furthermore, Jakobiec et al. [11] showed negative staining of adipophilin in a fibrous histiocytoma without a foamy cytoplasm, in contrast to our case, which contained mostly CD163 positive histiocytic cells with a foamy cytoplasm that stained strongly positive with adipophilin. Thus, both the histological and immunohistochemical profile of our patient's lesion indicate that it is a rare variant of the xanthomatous subtype. JXG typically presents with multiple cutaneous eruptions in the head, neck, and upper trunk [2] and is composed of lipid histiocytes, chronic inflammatory cells, and in classical cases Touton giant cells, which have an external ring of lipid [1]. Our patient had no cutaneous lesions and no Touton or other multinucleated giant cells, but showed similar mononucleated histiocytes that stained positive for CD34, which has been reported as negative in JXG $[2,12]$.

In 1970, Grayson and Pieroni [13] reported a solitary xanthoma of the limbus in an 11-year-old boy with histo- 
Fig. 1. Perilimbal conjunctiva and adjacent sclera lesion on left eye with ultrasound biomicroscopic (UBM) findings. a Inferotemporal, elevated yellow conjunctival lesion abutting the limbus OS. b UBM examination shows the lesion is $3.94 \mathrm{~mm}$ horizontally by $1.06 \mathrm{~mm}$ vertically extending to within $0.5 \mathrm{~mm}$ into the anterior chamber. c, d At 1 year, normal appearance and UBM imaging.

Fig. 2. Histopathological and immunohistopathological examination showed lipidcontaining histiocytes with sparse fibrous stroma. a The lesion extends to the deep surgical margin (arrow). b Vacuolated cells show no atypia or mitoses. Hematoxylineosin, original magnification $\times 100$ (a), $\times 400$ (b). c Adipophilin stain positive for intracytoplasmic lipid vacuoles (white boxes). d CD163 stain positive for macrophages. Original magnification $\times 1,000$ (c), $\times 400$ (d).
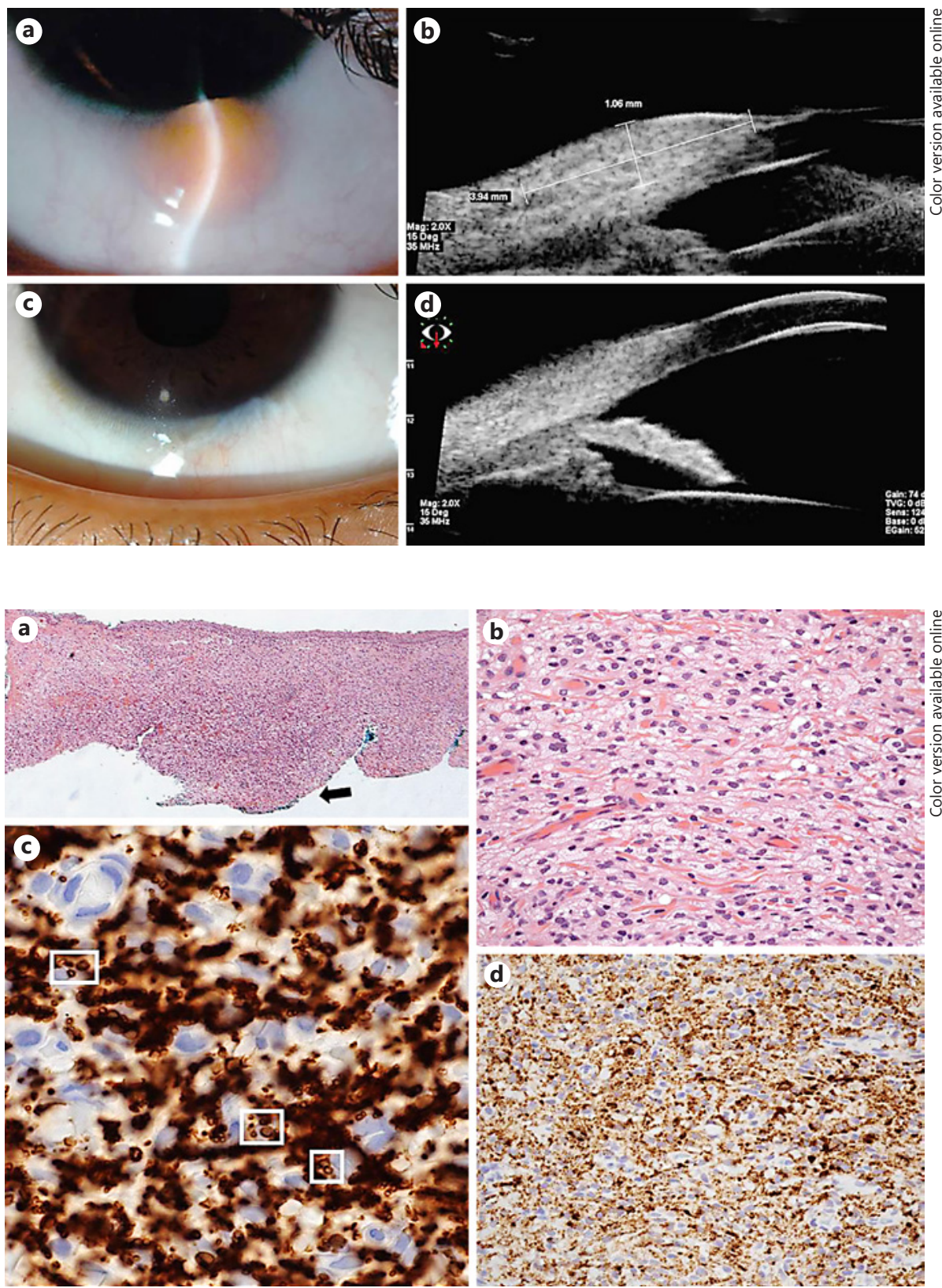

pathological similarities to our patient's lesion, consisting of foamy macrophages, but immunohistochemical studies were not yet available. However, their lesion contained prominent blood vessels and infiltrated the adjacent corneal stroma, obstructing the pupil. Our patient's lesion differed by predominately involving the perilimbal conjunctiva and sclera, containing only small blood vessels. In addition, we confirmed with immunohistochemical stains that the xanthomatous mass was composed predominantly of lipid-filled histiocytes.

Fibrous Histiocytoma of Xanthomatous Subtype
Furthermore, a review of 290 cases of benign fibrous histiocytomas of the skin included 10 (3.4\%) cases of "xanthomatous histiocytoma" subtype that showed similar histopathological features as in our case; these lesions were comprised of numerous foamy cells with relatively thin collagen bundles, rarely with a storiform pattern [5].

Thus, the lesion presenting in our patient demonstrates the spectrum of findings in fibrous histiocytoma in that our patient's lesion had a unique presentation of a

Ocul Oncol Pathol 2018;4:341-344 
xanthomatous subtype of the conjunctiva and sclera while the predominantly fibrous subtype occurs more commonly $[4,5]$.

Also, our patient's lesion may represent a choristoma as the history indicates a stable lesion involving the deep margins and possibly was present since birth.

We believe that patients with fibrous histiocytomas, whether fibrous or xanthomatous, should undergo lifetime follow-up examinations, as the risk of recurrent disease or transformation to a more aggressive condition is possible $[4,6]$.

\section{Statement of Ethics}

The patient and his parents have given informed consent for the publication of the clinical photographs and the medical findings. The institution review board of the Ottawa Hospital waived ethics review because this was a single case report that conformed to the Code of Ethics of the World Medical Association (Declaration of Helsinki) and informed patient consent was obtained.

\section{Disclosure Statement}

The authors have no financial conflict of interest to disclose.

\section{References}

1 Shields JA, Shields CL: Eyelid, Conjunctival, and Orbital Tumors: An Atlas and Textbook. Wolters Kluwer, 2007, vol 1, pp 675-690.

-2 Iwuagwu FC, Rigby HS, Payne F, Reid CD: Juvenile xanthogranuloma variant: a clinicopathological case report and review of the literature. Br J Plast Surg 1999;52:591-593.

-3 Gündüz K, Palamar M, Parmak N, Kuzu I: Eosinophilic granuloma of the orbit: report of two cases. J AAPOS 2007;11:506-508.

4 Font RL, Hidayat AA: Fibrous histiocytoma of the orbit. A clinicopathologic study of 150 cases. Hum Pathol 1982;13:199-209.

5 Gonzalez S, Duarte I: Benign fibrous histiocytoma of the skin. A morphologic study of 290 cases. Pathol Res Pract 1982;174:379-391.
6 Kim HJ, Shields CL, Eagle RC, Shields JA: Fibrous histiocytoma of the conjunctiva. Am J Ophthalmol 2006;142:1036-1043.

7 Lahoud S, Brownstein S, Laflamme MY: Fibrous histiocytoma of the corneoscleral limbus and conjunctiva. Am J Ophthalmol 1988; 106:579-583.

8 Soon AK, Brownstein S, O'Connor M, Iordanous Y: Fibrous Histiocytoma of the Conjunctiva. Ophthal Plast Reconstr Surg 2017; 33:e133.

9 Belliveau MJ, Brownstein S, Jordan DR, Faraji H: Low-grade, aggressive fibrous histiocytoma of the medial canthus. Can J Ophthalmol 2008;43:250.
0 Geoffrey R, Glasson B, Foster A: Benign fibrous histiocytoma of the conjunctiva. Case Rep Ophthalmol Med 2012:2012. DOI: $10.1155 / 2012 / 786260$

11 Jakobiec FA, Rai R, Yoon MK: Fibrous histiocytoma of the tarsus: clinical and immunohistochemical observations with a differential diagnosis. Cornea 2014;33:536-539.

12 Kraus M, Haley J, Ruiz R, Essary L, Moran C, Fletcher C: "Juvenile" xanthogranuloma: an immunophenotypic study with a reappraisal of histogenesis. Am J Dermatopathol 2001;23: 104-111.

13 Grayson M, Pieroni D: Solitary xanthoma of the limbus. Br J Ophthalmol 1970;54:562564 . 